\title{
THE REACTIONS OF THE «BEAM - FOUNDATION» SYSTEM TO THE SUDDEN CHANGE OF THE BOUNDARY CONDITIONS
}

\author{
Vladimir A. Gordon ${ }^{1}$, Olga V. Pilipenko ${ }^{1}$ and Vladimir A. Trifonov ${ }^{2}$ \\ ${ }^{1}$ Orel State University, Orel, 302026, Russia \\ ${ }^{2}$ Gazprom-socinvest, St. Petersburg, Russia
}

\begin{abstract}
The authors constructed a mathematical model of a dynamic process in a loaded beam on the elastic Winkler foundation in a sudden formation of a defect in the form of a change in the boundary conditions. The solution of the static problem of bending of the beam pinched at the ends served as the initial condition for the process of forced vibrations hinged supported at the ends of a beam, which arose after a sudden break in the connections that prevented the rotation of the end sections. The authors determined the dynamic increments of stresses in a beam for various combinations of a beam and foundation parameters.
\end{abstract}

\section{INTRODUCTION}

An important problem of construction mechanics is the analysis of the sensitivity of load-bearing structures to structural rearrangements under load such as suddenly disconnected connections, cracks, fractures, etc. Obtaining such information for real constructions requires the development of special methods, since this problem cannot be solved by universal methods. From the standpoint of structural mechanics in these problems, it becomes necessary to calculate such systems as constructively nonlinear, changing the design scheme under load, i.e. with dynamic overloads, caused by sudden beyond projected effects.

In the present work, the task is to construct a mathematical model of transient dynamic processes in a beam on an elastic foundation when a defect is suddenly formed in the form of a change in the boundary conditions. Before the formation of a defect, the reaction of the structure is determined by a static action. The sudden formation of a defect leads to a reduction in the overall rigidity of the structure, which does not ensure the static equilibrium of the system. The inertial forces that have arisen cause a dynamic reaction, redistribution and growth of strains and stresses. As a result, there may be a violation of the regular functioning of the structure, or loss of load capacity and destruction.

\section{STATEMENT OF THE PROBLEM}

The elastic beam with flexural stiffness $E I$ rests on the entire length $l$ on the elastic Winkler foundation with stiffness coefficient $k$, rigidly clamped at the ends. The uniformly distributed intensity load $q$ and the foundation reaction affect the outer layers of the beam. It is assumed that at some point in time $t=0$, the connections in a statically deformed beam, which prevent the rotation of the end sections in the supports, suddenly collapsed, forming hinges in the place of sealing. The static state of the loaded beam ceases to be in equilibrium and the beam will move into motion $v(x, t)$, during which the deformations and stresses in the beam acquire dynamic increments. 


\section{SOLUTION OF THE PROBLEM}

The problem is solved in the following sequence:

1) we determine the static deflection of a ("undamaged") beam with clamped ends on an elastic foundation, which is used subsequently as the initial condition of a dynamic process, which is initiated in the system by a sudden transformation of the boundary conditions;

2) we determine the frequencies and forms of bending vibrations of a ("damaged") beam with hinged ends on an elastic foundation;

3 ) we study the forced bending vibrations of a loaded beam. In this case, the load, the static deflection of the "undamaged" beam and the desired dynamic deflection are decomposed into series according to the modes of natural vibrations of the "damaged" beam.

3.1 The static bending of a beam, which is pinched at the ends on Winkler foundation, in dimensionless variables and parameters

$$
\xi=\frac{x}{l}, \quad w_{s t}=\frac{v}{l}, \quad \bar{q}=\frac{q l^{3}}{E I}, \quad \alpha=\sqrt[4]{\frac{k l^{4}}{4 E I}} .
$$

is described by equation $[1,2]$

$$
\frac{d^{4} w_{s t}}{d \xi^{4}}+4 \alpha^{4} w_{s t}=\bar{q}
$$

The general solution of equation (1) in the case of pinching ends has the form $[1,2]$

$$
w_{s t}=\frac{\bar{q}}{4 \alpha^{4}}\left(1-k_{4}(\alpha \xi)\right)+w_{0}^{\prime \prime} k_{2}(\alpha \xi)+w_{0}^{\prime \prime \prime} k_{1}(\alpha \xi)
$$

where $k_{i}(\alpha \xi)(i=1 \div 4)$ is Krylov function of the form

$$
k_{1}(\alpha \xi)=\frac{\sin \alpha \xi \operatorname{ch} \alpha \xi-\cos \alpha \xi \operatorname{sh} \alpha \xi}{4 \alpha^{3}}, \quad k_{2}=k_{1}^{\prime}, \quad k_{3}=k_{2}^{\prime}, \quad k_{4}=k_{3}^{\prime}
$$

$w_{0}^{\prime \prime}, w_{0}^{\prime \prime \prime}$ are the initial parameters, respectively, the dimensionless bending moment and the shear force at the origin $\xi=0$.

The dimensionless bending moment in a static state is determined by the function

$$
w_{s t}^{\prime \prime}=\bar{q} k_{2}(\alpha \xi)+w_{0}^{\prime \prime} k_{4}(\alpha \xi)+w_{0}^{\prime \prime \prime}(\alpha \xi) \text {. }
$$

Figure 1 shows the diagrams of bending moments in a beam with clamped ends for various values of the generalized rigidity of the "beam-foundation" system $\lambda=4 \alpha^{4}$. It is worth paying attention to the somewhat "unusual" form, which takes the moment epures with increasing rigidity of the system - the moments in the central part of the beam are much lower than in the quarter of spans. This is the result of the combined effect of external unloading on the beam and the reaction of the elastic foundation.

$$
\lambda=10^{3,5}
$$

$$
\lambda=10^{4,5}
$$
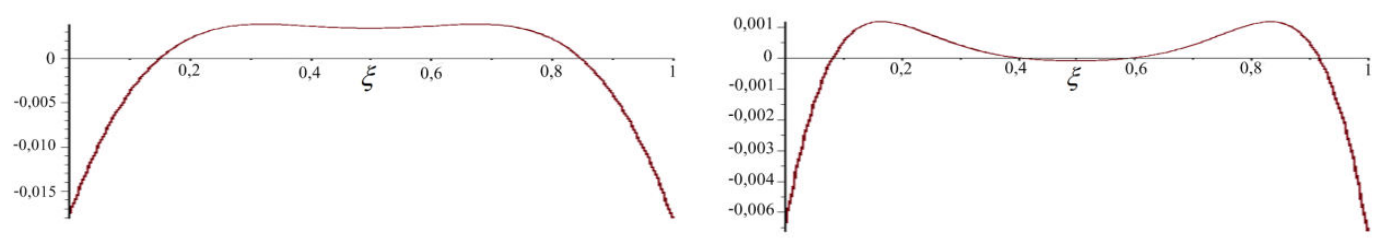

Figure 1: Epures of bending moments in the initial static state, depending on the rigidity index of the "beam-foundatio" system $\lambda$ 
3.2 The resulting motion $v_{d y n}=v(x, t)$ after a sudden transformation of the restraints of the beam into the hinges is described by equation [2]

$$
\frac{\partial^{4} w_{d y n}}{\partial \xi^{4}}+4 \alpha^{4}\left(w_{d y n}+\frac{\partial^{2} w_{d y n}}{\partial \tau^{2}}\right)=\bar{q},
$$

where $w_{d y n}=\frac{v(x, t)}{l}, \tau=w_{0} t . w_{0}=\sqrt{\frac{k}{\rho A}}$ is a parameter having a frequency

dimension and, therefore, called a "conventional" frequency.

Equation (4) describes the forced vibrations of a loaded beam. The Winkler model does not imply dynamic phenomena in the elastic foundation. The required eigenfunctions and frequencies of the problem will be obtained from equation (4) with the zeroed right-hand side, which after separation of the variables by representation

$$
w_{d y n}=W(\xi) \sin \bar{\omega} \tau
$$

takes the form

$$
\frac{d^{4} W}{d \xi^{4}}+4 \alpha^{4}\left(1-\bar{\omega}^{2}\right) W=0
$$

where $\bar{\omega}=\frac{\omega}{\omega_{0}}$ is a dimensionless eigenfrequency of the bending vibrations of a beam on an elastic foundation.

Using the "conventional" frequency $\omega_{0}$, characterizing stiff and inertial properties of the "beam-foundation" system, and the known basic frequency of bending vibrations of a free beam supported in the same way (without foundation support)

we bring the equation to the form (6)

$$
\omega_{1 f}=\left(\frac{\pi}{l}\right)^{2} \sqrt{\frac{E I}{\rho A}}
$$

$$
\frac{d^{4} W}{d \xi^{4}}=\pi^{4}\left(-\bar{\omega}_{0}^{2}+82\right) W
$$

where $\bar{\omega}_{0}=\frac{\omega_{0}}{\omega_{1 f}}$ is a relative "conventional" frequency; $\not \sigma=\frac{\omega}{\omega_{1 f}}$ is a relative required frequency.

Using Euler substitution

$$
W=A e^{r \xi}
$$

we find the characteristic equation for differential equation (7)

$$
r^{4}+\pi^{4}\left(\bar{\omega}_{0}^{2}-\phi^{2}\right)=0
$$

roots of which can be represented in two ways, depending on the ratios of frequencies $\bar{\omega}_{0}$ and $\mathscr{c}$ : 
1) if $\$ 8 \bar{\omega}_{0}$, then the roots of equation (9) are real and purely imaginary

$$
r_{1,2}= \pm \beta_{1}, \quad r_{3,4}= \pm i \beta_{1}, \quad \beta_{1}=\pi \sqrt[4]{8^{2}-\bar{\omega}_{0}^{2}},
$$

the deflection function (8) has the form

$$
W=A_{1} \operatorname{ch} \beta_{1} \xi+A_{2} \operatorname{sh} \beta_{1} \xi+A_{3} \cos \beta_{1} \xi+A_{4} \sin \beta_{1} \xi ;
$$

2) if $\$ 2<\bar{\omega}_{0}$, then the roots of equation (9) are complex

$$
r_{1,2,3,4}=( \pm i \pm 1) \beta_{2}, \quad \beta_{2}=\frac{\pi}{\sqrt{2}} \sqrt[4]{\bar{\omega}^{2}-3^{2}}
$$

and the deflection function

$$
\begin{gathered}
W=A_{1} \operatorname{sh} \beta_{2} \xi \sin \beta_{2} \xi+A_{2} \operatorname{sh} \beta_{2} \xi \cos \beta_{2} \xi+ \\
+A_{3} \operatorname{ch} \beta_{2} \xi \sin \beta_{2} \xi+A_{4} \operatorname{ch} \beta_{2} \xi \cos \beta_{2} \xi .
\end{gathered}
$$

It was shown in $[3,4]$ that for a beam completely supported on the Winkler foundation, in the case of canonical boundary conditions - pinch- pinch, pinch - hinge, hinge-hinge, console - only variant (10) is realized.

Using the initial parameters

$$
W_{0}=W(0), \quad W_{0}^{\prime}=W^{\prime}(0), \quad W_{0}^{\prime \prime}=W^{\prime \prime}(0), \quad W_{0}^{\prime \prime \prime}=W^{\prime \prime \prime}(0)
$$

instead of integration constants $A_{i}(i=1 \div 4)$, we write the relations characterizing the state of arbitrary cross-section $\xi$ of the beam, using version (10), (11).

In this case, the deflection function has the form

$$
W(\xi)=W_{0} R_{4}\left(\beta_{1} \xi\right)+W_{0}^{\prime} R_{3}\left(\beta_{1} \xi\right)+W_{0}^{\prime \prime} R_{2}\left(\beta_{1} \xi\right)+W_{0}^{\prime \prime \prime} R_{1}\left(\beta_{1} \xi\right),
$$

where $R_{i}(i=1 \div 4)$ - Krylov functions of the form

$$
R_{1}\left(\beta_{1} \xi\right)=\frac{s h \beta_{1} \xi-\sin \beta_{1} \xi}{2 \beta_{1}^{3}} ; \quad R_{2}=R_{1}^{\prime} ; \quad R_{3}=R_{2}^{\prime} ; \quad R_{4}=R_{3}^{\prime} .
$$

The state of the arbitrary beam section is described by the matrix equation

$$
\bar{W}(\xi)=V_{1}(\xi) \bar{W}_{0},
$$

where $\bar{W}(\xi)$ is a state vector of arbitrary section $\xi$

$$
\bar{W}(\xi)=\left\{W(\xi) W^{\prime}(\xi) W^{\prime \prime}(\xi) W^{\prime \prime \prime}(\xi)\right\} ;
$$

$\bar{W}_{0}(\xi)$ is a vector of initial parameters

$$
\bar{W}_{0}=\left\{\begin{array}{llll}
W_{0} & W_{0}^{\prime} & W_{0}^{\prime \prime} & W_{0}^{\prime \prime \prime}
\end{array}\right\} ;
$$

$V_{1}(\xi)=\left\{v_{i j}\right\}$ is a functional matrix of influence of initial parameters on the state of section $\xi$ 


$$
V_{1}(\xi)=\left(\begin{array}{cccc}
R_{4}\left(\beta_{1} \xi\right) & R_{3}\left(\beta_{1} \xi\right) & R_{2}\left(\beta_{1} \xi\right) & R_{1}\left(\beta_{1} \xi\right) \\
\beta_{1}^{4} R_{1}\left(\beta_{1} \xi\right) & R_{4}\left(\beta_{1} \xi\right) & R_{3}\left(\beta_{1} \xi\right) & R_{2}\left(\beta_{1} \xi\right) \\
\beta_{1}^{4} R_{2}\left(\beta_{1} \xi\right) & \beta_{1}^{4} R_{1}\left(\beta_{1} \xi\right) & R_{4}\left(\beta_{1} \xi\right) & R_{3}\left(\beta_{1} \xi\right) \\
\beta_{1}^{4} R_{3}\left(\beta_{1} \xi\right) & \beta_{1}^{4} R_{2}\left(\beta_{1} \xi\right) & \beta_{1}^{4} R_{1}\left(\beta_{1} \xi\right) & R_{4}\left(\beta_{1} \xi\right)
\end{array}\right) .
$$

3.3 We will conduct an analysis of free frequencies and forms of flexural vibrations of a beam on an elastic foundation when the ends are hinged. In this case, the boundary conditions and the deflection function have the form

$$
\begin{gathered}
W_{0}=W_{0}^{\prime \prime}=0, \\
W(1)=W^{\prime \prime}(1)=0 . \\
W(\xi)=W_{0}^{\prime} R_{3}\left(\beta_{1} \xi\right)+W_{0}^{\prime \prime \prime} R_{1}\left(\beta_{1} \xi\right) .
\end{gathered}
$$

Satisfying the second pair of boundary conditions (16), from function (17) and its second derivative, we obtain a system of two algebraic equations of relatively unknown initial parameters and $W_{0}^{\prime}$ и $W_{0}^{\prime \prime \prime}$

$$
\left\{\begin{array}{l}
W_{0}^{\prime} R_{3}\left(\beta_{1}\right)+W_{0}^{\prime \prime \prime} R_{1}\left(\beta_{1}\right)=0, \\
W_{0}^{\prime} \beta_{1}^{4} R_{1}\left(\beta_{1}\right)+W_{0}^{\prime \prime \prime} R_{3}\left(\beta_{1}\right)=0 .
\end{array}\right.
$$

Equating the determinant of the system (18) to zero, we obtain the frequency equation

$$
4 \sin \beta_{1} \operatorname{sh} \beta_{1}=0 \text {, }
$$

whence it follows that

$$
\beta_{1 n}=n \pi(n=1,2,3, \ldots) .
$$

Taking into account formula (10), we obtain the frequency spectrum

$$
\phi_{h}=\sqrt{\bar{\omega}_{0}^{2}+n^{4}}
$$

From any equation of the system (18) with $\beta_{1 n}=n \pi$, it follows that

$$
\frac{W_{0}^{\prime \prime \prime}}{W_{0}^{\prime}}=-(n \pi)^{2},
$$

then, according to (17), the $n$-th form with frequency $\bar{\omega}_{n}$, has the form

$$
W_{n}(\xi)=A_{n} \sin n \pi \xi
$$

where $n$ is a number of half-waves of a sinusoid on length $l$ of a beam;

$A_{n}$ is an unknown amplitude of vibrations in $n$ - $t$ th form.

Thus, the forms of free vibrations of the beam on an elastic foundation remain the same as for the free beam, but with frequencies

$$
\omega_{n}=\sqrt{\bar{\omega}_{0}^{2}+n^{4}} \omega_{n f} .
$$

3.4 The solution of the differential equation of forced vibrations (4) will be sought by expanding the function $w_{d y n}(\xi, \tau)$ in a series of eigenfunctions $W_{n}(\xi)$ (21) with coefficients in the form of unknown time functions $Q_{n}(\tau)$ 


$$
w_{d y n}=\sum_{n=1}^{\infty} Q_{n}(\tau) W_{n}(\xi) .
$$

We find functions $Q_{n}(\tau)$ using the following procedures: substituting the series (13) and the expression (2) into equation (4), multiplying both sides of this equation by $W_{n}(\xi)$, integrating both parts of $\xi$ from 0 to 1 and, using the property of orthogonality of the modes of free vibrations $W_{n}(\xi)$, we obtain the differential equation for defining functions $Q_{n}(\tau)$

$$
\frac{d^{2} Q_{n}}{d \tau^{2}}+\bar{\omega}_{n}^{2} Q_{n}=\frac{2 \bar{q}}{\pi^{4} \bar{\omega}_{0}^{2}} \frac{\sin ^{2} \frac{n \pi}{2}}{\frac{n \pi}{2}}=S_{n},
$$

The general solution of the inhomogeneous equation (23)

$$
Q_{n}=D_{1 n} \cos \frac{\phi_{h}}{\bar{\omega}_{0}} \tau+D_{2 n} \sin \frac{\phi_{h}}{\bar{\omega}_{0}} \tau+\frac{S_{n}}{\bar{\omega}_{n}^{2}}
$$

is a sum of the solution of the relevant homogeneous equation (the first two terms) and the particular solution corresponding to the right-hand side of (23) (the third term).

Now, according to (22), the dynamic deflection function takes the form

$$
w_{d y n}=\sum_{n=1}^{\infty}\left(D_{1 n} \cos \frac{\Phi_{h}}{\bar{\omega}_{0}} \tau+D_{2 n} \sin \frac{\Phi_{h}}{\bar{\omega}_{0}} \tau+\frac{S_{n}}{\bar{\omega}_{n}^{2}}\right) \sin n \pi \xi .
$$

The constants of integration $D_{1 n}$ and $D_{2 n}$ are determined from the initial conditions

$$
\begin{gathered}
w_{d y n}(\xi, 0)=w_{c m}(\xi),\left.\quad \frac{\partial w_{d y n}}{\partial \tau}\right|_{\xi, 0}=0 . \\
D_{1 n}=2 \int_{0}^{1} w_{s t}(\xi) \sin n \pi \xi d \xi-\frac{S_{n}}{\bar{\omega}_{n}^{2}}=B_{n}-\frac{S_{n}}{\bar{\omega}_{n}^{2}}, \quad D_{2 n}=0 .
\end{gathered}
$$

Substituting (27) into (25), and taking into account the trigonometric identity

we get

$$
1-\cos \frac{B_{h}}{\bar{\omega}_{0}} \tau=2 \sin ^{2} \frac{B_{h}}{2 \bar{\omega}_{0}} \tau,
$$

$$
w_{d y n}=\sum_{n=1}^{\infty}\left(B_{n} \cos \frac{\phi_{h}}{\bar{\omega}_{0}} \tau+C_{n} \sin ^{2} \frac{\Phi_{h}}{2 \bar{\omega}_{0}} \tau\right) \sin n \pi \xi,
$$

where $C_{n}=\frac{4 \bar{q}}{\pi^{4} \phi_{\overparen{h}}^{2}} \frac{\sin ^{2} \frac{n \pi}{2}}{\frac{n \pi}{2}}$. 
The dimensionless bending moment is obtained by differentiating the series (28) twice

$$
w_{d y n}^{\prime \prime}=-\pi^{2} \sum_{n=1}^{\infty} n^{2}\left(B_{n} \cos \frac{\phi_{h}}{\bar{\omega}_{0}} \tau+C_{n} \sin ^{2} \frac{\phi_{h}}{2 \bar{\omega}_{0}} \tau\right) \sin n \pi \xi \text {. }
$$

\section{A NUMERICAL EXAMPLE}

The Maple software package helped to calculate the dimensionless deflections $w(\xi)$ and bending moments $w^{\prime \prime}(\xi)$ in a beam, which is loaded by a uniformly distributed intensity load $\bar{q}=1$, on the elastic Winkler foundation

- in an initial static state, when the ends are pinched: $w_{s t}(\xi), w_{s t}^{\prime \prime}(\xi)$;

- in a static state formed after a quasi-static transformation of pinches into hinges $w_{q}(\xi), w_{q}^{\prime \prime}(\xi)$

- in a dynamic process that occurs when in a sudden transformation of pinches into hinges: $w_{d y n}(\xi, \tau), w_{d y n}^{\prime \prime}(\xi)$.

In practical calculations, 20 series members (19) and (20) were taken into account. In this case, we obtain a practical coincidence of epures of the dynamic deflection $w_{d y n}(\xi, 0)$ and the static deflection $w_{s t}(\xi)$, that is,

$$
\sum_{n=1}^{20} B_{n} \sin n \pi \xi \approx w_{s t}(\xi)
$$

The results of the calculations are shown in Figures 2 and 3, as well as in Table 1. In Figures 2 and 3 are shown respectively: epures of bending moments $w_{q}^{\prime \prime}(\xi)$ in a beam after quasi-static of transformation of pinches into hinges, and during vibrations $w_{d y n}^{\prime \prime}\left(\xi, \tau_{0}\right)$ after sudden transformation of pinches into hinges at the time $\tau_{0}$ of reaching the highest values. The epures are constructed for different values of the stiffness parameter of the "beam-foundation" system $\lambda$.

$$
\lambda=10^{3,5}
$$

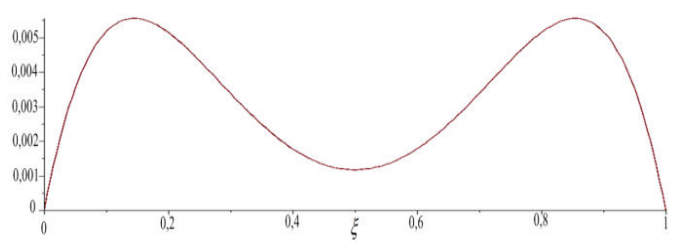

$$
\lambda=10^{5}
$$

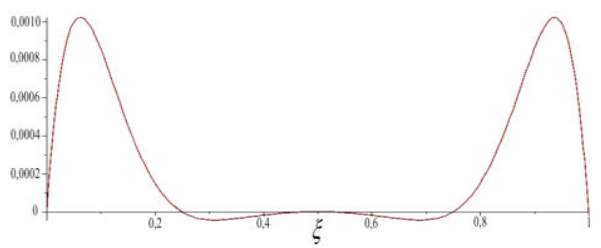

Figure 2: Epures of bending moments after quasi-static transformation of boundary conditions depending on the rigidity index of the "beam-foundation" system $\lambda$

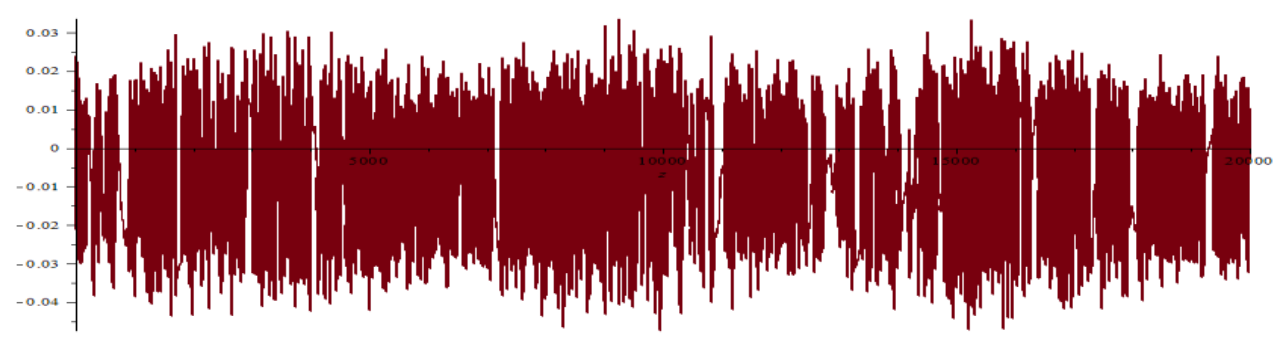

Figure 3: Moments in the cross section $\xi=0,16$ (time scanning). $M_{\max }=0,047$ 
Table 1: Influence of the rigidity of the "beam-foundation" system on the increment of bending moments

\begin{tabular}{|c|c|c|c|c|c|}
\hline$\lambda$ & $\begin{array}{c}w_{s t}^{\prime \prime} \\
\max \end{array}$ & $\begin{array}{c}w_{q}^{\prime \prime} \\
\max \end{array}$ & $K_{s t}$ & $w_{d y n}^{\prime \prime}$ & $K_{d y n}$ \\
\hline 0 & 0,083 & 0,125 & 1,506 & 0,3 & 3,614 \\
\hline 10 & 0,082 & 0,112 & 1,366 & 0,269 & 3,28 \\
\hline $10^{1,5}$ & 0,079 & 0,093 & 1,177 & 0,225 & 2,848 \\
\hline $10^{2}$ & 0,071 & 0,06 & 0,845 & 1,156 & 2,197 \\
\hline $10^{2,5}$ & 0,0544 & 0,0265 & 0,487 & 0,07 & 1,287 \\
\hline $10^{3}$ & 0,0334 & 0,0104 & 0,311 & 0,0346 & 1,036 \\
\hline $10^{3,5}$ & 0,018 & 0,0055 & 0,305 & 0,0186 & 1,033 \\
\hline
\end{tabular}

\section{CONCLUSIONS}

If we consider the transformation of the boundary conditions in the given "beamfoundation system" under the load as a defect, then the conducted study shows that the quasi-static formation of a defect, that is, a reduction in the rigidity of the end supports, leads to an insignificant increase in the maximum stresses in the beam $\left(K_{s t}>1\right)$ if there is no foundation $(\lambda=0)$ and low values of the indicator of the "beam-foundation" system $\left(0<\lambda \leq 10^{1,79}\right)$. For beams resting on more rigid foundations $\left(\lambda>10^{1,79}\right)$, the formation of the same defect, on the contrary, leads to a decrease in the greatest stresses $\left(K_{s t}<1\right)$.

A sudden formation of a defect gives more than three times $\left(K_{d y n}=3,614\right)$ an increase in the maximum stress in a free beam $(\lambda=0)$. For systems with higher rigidity, the effect of transforming the boundary conditions is reduced. There is a redistribution of stresses along the span, but the greatest stress at $\lambda>10^{4}$ does not exceed the initial static value $\left(K_{d y n}=1\right)$. In addition, regardless of the rate of defect formation with increasing rigidity of the system, the greatest stresses move from the center of the beam to the periphery of the span.

The work was conducted within the framework of the basic part of the state task 1.5265.2017/BP (1.5265.2017/8.9)

\section{References}

1. V. Gordon, O. Pilipenko, Proceedings of the 22 Intern. Congress on Sound and Vibration. Florence, Italy, (2015)

2. V. Gordon, O. Pilipenko, Proc. European Congress on Computational Methods in Applied Sciences and Engineering, 3. Crete, Greece, (2016)

3. V. Gordon, E. Bruma, T. Poturaeva, Fundamental and Applied Problems of Engineering and Technology, 1 (2017)

4. V. Gordon, E. Bruma, Vibration technologies, mechatronics and controlled machines: collection of scientific articles in 2 parts. South-West State University. Kursk (2016) 